\title{
PREPARACIÓN PARA LA JUBILACIÓN EN LOS SERVICIOS PÚBLICOS DE CHILE
}

\section{RETIREMENT PREPARATION FOR THE PUBLIC SERVICE OF CHILE}

\author{
Marlene Araya Cuello 1 \\ Luzmarina Silva Concha ${ }^{2}$
}

\section{Resumen.}

La jubilación supone una ruptura en la vida de los individuos, el paso de una forma de vida centrada en el trabajo, el cual ha sido predominante en la vida, a otra nueva etapa que anuncia el comienzo de la vejez y que exige al individuo adaptaciones, tanto en su vida como en sus propias expectativas.

El objetivo de este estudio es conocer las percepciones y las medidas tomadas por los trabajadores (ras) prejubilables del Instituto de Previsión Social ex INP (IPS organismo público de seguridad social) y el Servicio de Registro Civil e Identificación, sobre la jubilación, en las comunas de Valparaíso, Viña del Mar, Quilpué, San Felipe, Los Andes y el significado que le atribuyen al trabajo, además de saber si estas percepciones están influenciadas por el género o escalafón social al cual pertenecen.

Es un estudio exploratorio descriptivo, que utiliza, en la recolección de datos, tanto técnicas cuantitativas como cualitativas. Existe en todos los estamentos (profesionales, administrativos) e indistintamente del sexo hombre o mujer, una alta valoración hacia el trabajo. Solo la mitad de los encuestados ha planificado su tiempo una vez que jubile... Los entrevistados no han tomado medidas para su jubilación, en el ámbito económico y social. Hay escasez de relaciones sociales fuera del ámbito laboral, lo cual influirá negativamente a futuro en el proceso de adaptación de la persona mayor a su bienestar subjetivo y calidad de vida.

Palabras Claves: Jubilación, Preparación para la jubilación, Trabajo, Instituto de Previsión Social.

\begin{abstract}
s
Retirement is a break in the lives of individuals, the transition from one way of living centred on work, which has been dominant throughout his/her life, to a new stage that announces the beginning of old age and requires the individual's adaptation, both in life and in their own expectations.

The aim of this study was to determine the perceptions and actions taken by employees in early retirement from the Social Security Agency, former INP (IPS public social security agency) and the Service of Civil Registry and Identification, in relation to retirement, in the communes of Valparaíso, Viña del Mar, Quilpué, San Felipe, Los Andes; and the meaning attributed to work, also whether these perceptions are influenced by gender or scaffold to which they belong.

It is an exploratory-descriptive study, using quantitative and qualitative techniques in data collection. It exists at all levels (professional, administrative) and either male or female, a high valuation to work. Only half of the respondents planned their time once they retire ... Respondents have not taken steps to their retirement in the economic and social fiel. There is a shortage of social relationships outside work, which

\footnotetext{
${ }^{1}$ Asistente Social, Magister en Gerontología Social, académica Universidad de Valparaíso, Carrera de Trabajo Social,

${ }^{2}$ Terapeuta ocupacional, Magister en Gerontología Social, académica, Universidad de Playa Ancha, Carrera de Terapia Ocupacional. Fonos 032-2500235 032-2205372 .lsilvaconcha@gmail.com
} 
will negatively affect the future in the process of adaptation of the older person to their subjective wellbeing and quality of life.

Key words: Retirement, Preparation For Retirement, Work, Social security agency

\section{INTRODUCCIÓN}

De acuerdo a los datos arrojados por el censo del año 2002 en nuestro país, el total de la población de 60 años y más experimentó un crecimiento absoluto de 411.487 personas, pasando de 1.305.557 a 1.717.478 adultos mayores, con una tasa de crecimiento anual muy superior al total del país. (1)

Los datos expuestos sobre el envejecimiento poblacional, nos llevan a meditar sobre el peso que tiene para la sociedad este sector en constante aumento, ya que significaría un incremento en la demanda de bienes y servicios, lo que se traducirá en una mayor saturación del sistema social y en una sobrecarga para los demás sectores de la población, produciéndose una segura modificación de los actuales sistemas, laborales, sanitarios, y educacionales.

La jubilación es una de las preocupaciones existentes en todas las sociedades, debido a que la población se encuentra cada día mas envejecida y el número de jubilados aumenta progresivamente. Las causas de esta preocupación se inscriben en la serie de dificultades, económicas, familiares, psicológicas, físicas y otras, que tienen lugar en esta etapa de la vida, en la que se presenta además, una notable disminución de la capacidad de adaptación influenciada por muchos factores culturales, sociales, de salud o de personalidad de cada uno de los adultos mayores. En Ciencia de la Ocupación la adaptación se define como la "respuesta interna a un desafío significativo en el desempeño de roles". (2) De esta manera, en la vida real no sólo las personas en riesgo de adquirir una discapacidad o aquellas que la presenten, tienen necesidad de adaptación, sino que de hecho todas las personas, continuamente, deben adaptarse de diversas formas en la organización y en la realización de sus rutinas diarias, siendo este periodo previo a la jubilación, donde se debe organizar esta transición o continuidad de roles.

"La jubilación implica un cambio social y económico y no existen mecanismos generales para este cambio, como en otras etapas de la vida, educación, primer empleo, etc. Cualquier cambio social se asume mejor a través de un período de preparación que permita un ajuste individual y social. Este período de transición ocupacional se realiza sin ninguna preparación específica, por lo que resulta lógico pensar que se debería preparar a los jubilables en la variedad de cambios sociales, económicos y familiares, que la jubilación conlleva." (3)

Muchos esperan el momento de la jubilación con ansias porque representará descanso y dedicación a sí mismos y a su familia, sin embargo, para otros resulta inimaginable concebirse sin trabajar y se les hace difícil aceptar que son prescindibles y reemplazables. Se resisten a la idea de pasar a ser parte de la población pasiva, que en nuestra cultura es sinónimo de improductividad.

La mayoría de los estudios sobre jubilación y preparación de la jubilación se centran en la población masculina, como una población homogénea sin tener en cuenta las necesidades individuales y la población femenina. Los estudios han demostrado que existen diferencias 
individuales que impiden concebir los cursos de preparación para la jubilación como verdaderas recetas. (4)

Cuando se trata de población masculina, se suele presentar la jubilación como una ruptura con la vida laboral, la que se acompaña de una crisis llamada "crisis de jubilación". (5) Para las mujeres, socialmente concebidas en los roles de esposa, madre, dueña de casa, se sigue considerando la pérdida de la actividad laboral, de modo general, como secundaria, que no necesariamente conlleva una ruptura penosa en el curso de la existencia.

En nuestro país existe un desconocimiento acerca de si los trabajadores (as) están preparando su tiempo de jubilación, de manera que se puedan crear programas de preparación para la jubilación de acuerdo a las necesidades de los actores sociales.

Esto nos lleva a plantearnos las siguientes interrogantes:

¿Cuál es la percepción que los trabajadores (as) prejubilables tienen de la jubilación?

¿Los trabajadores (as) prejubilables están tomando medidas para preparar su jubilación y tiempo libre. ?

\section{METODOLOGÍA}

La presente investigación es de tipo exploratorio-descriptiva, con un diseño no experimental transeccional descriptivo. (6) Las variables de estudio son: Percepción de la jubilación, Preparación de la jubilación en sus dimensiones: Salud, Economía, Redes y Relaciones Sociales y Tiempo Libre.

La población de estudio corresponde a funcionarios/as pertenecientes al INP y Registro Civil e Identificación de las comunas de Valparaíso, Viña del Mar, San Felipe, Quilpue, Quillota los cuales debían cumplir con los siguientes criterios de inclusión:

Funcionarios (as) pertenecientes al INP y/o al Registro Civil e Identificación de las comunas de Valparaíso, Viña del Mar, San Felipe, Quillota y Quilpue de 50 años de edad y más para las mujeres 55 años y más para los hombres. De esta forma, la población estudiada queda conformada por 107 funcionarios (as).

Instrumento de recolección de los datos:

Cuestionario de 84 preguntas cerradas, con alternativas, en el que se recolectan antecedentes generales, percepción frente a la jubilación y medidas tomadas para enfrentar la jubilación en relación a: salud, vivienda, economía, redes y relaciones sociales y tiempo libre. El instrumento fue creado especialmente para la investigación y se aplicó mediante auto administración durante un periodo de 2 meses. Fue validado para contenidos a través del juicio de expertos y luego se determinó su confiabilidad mediante prueba de estabilidad de Test re Test.

Los encuestados firmaron el consentimiento informado autorizando la utilización de los resultados con fines académicos manteniendo el anonimato de ellos. 


\section{RESULTADOS}

\section{Perfil de los encuestados.}

El 58\% pertenece al sexo femenino con una edad promedio de 54 años, estado civil casado con un $60 \%$, un $12 \%$ tanto para separados, como para solteros sin pareja, un $7 \%$ corresponde tanto a viudos y separados con pareja y un $2 \%$ solteros con pareja. Un $70 \%$ de ellos pertenece al escalafón técnico administrativo y un 30\% al directivo profesional, el mayor porcentaje de ellos comenzó a trabajar antes de los 20 años de edad (55\%), destacándose el tramo entre los 18 y los 20 con un $41 \%$ y entre los 21 y 23 años con un $23 \%$. Un 65\% pertenece a FONASA y $35 \%$ a ISAPRE. Respecto del sistema de pensiones se reparte 50\% para AFP y 50\% INP. Un 55\% no padece de patologías crónicas en tanto el $45 \%$ si las padece.

\section{VARIABLE ESTUDIO 1. PERCEPCIÓN FRENTE A LA JUBILACIÓN.}

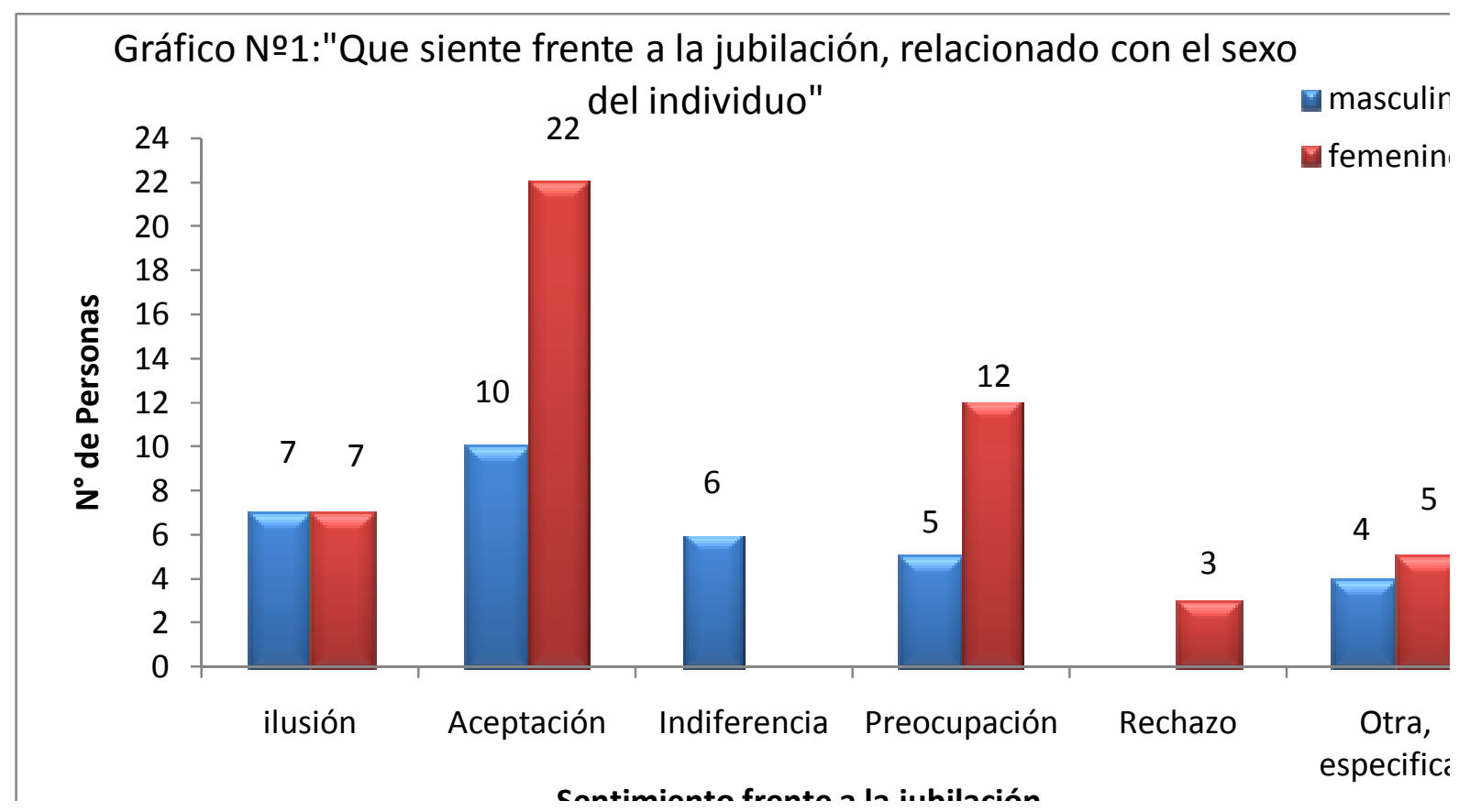

\section{Grafico $\mathbf{N}^{0} 1$ ¿Qué siente ante la jubilación?}

En cuanto a la pregunta: ¿Que siente usted frente a la jubilación?, relacionado con el sexo del individuo, 32 personas (37\%) la acepta, en el que un $69 \%$ corresponde al sexo femenino y el $31 \%$ restante corresponde al sexo masculino. En la segunda mayoría tenemos que a 17 personas (21\%) les preocupa, lo que se distribuye en un $70 \%$ sexo femenino y un $30 \%$ sexo masculino. A 14 personas (17\%) le provoca ilusión, repartiéndose por igual porcentaje en ambos sexos.

En cuanto a la pregunta: ¿Que siente usted frente a la jubilación?, relacionada con el escalafón del individuo, en el 37\% que la acepta, 69\% corresponde al escalafón Administrativo-Técnico y el $31 \%$ restante corresponde a Directivo-Profesional. En la segunda mayoría a un $21 \%$ le preocupa, distribuido en un 57\% Administrativo-Técnico y un 43\% Directivo-Profesional. En 
cuanto a si le produce algún grado de incertidumbre saber que algún día tendrá que jubilar, el

Tipo de Incertidumbre

\begin{tabular}{|l|c|c|}
\hline \multicolumn{1}{|c|}{ Tipo de Incertidumbre } & Frecuencia & Porcentaje \\
\hline Económica & 35 & 42 \\
\hline Económica y llegar a la tercera edad & 20 & 24 \\
\hline Llegar a al tercera edad & 17 & 20 \\
\hline Falta de actividad laboral & 6 & 7 \\
\hline Exceso de tiempo libre & 2 & 2,5 \\
\hline Económica, exceso de tiempo libre y llegar a la tercera edad & 2 & 2,5 \\
\hline Económica y exceso de tiempo libre & 1 & 1 \\
\hline Económica y falta de actividad laboral & 1 & 1 \\
\hline Total & $\mathbf{8 4}$ & $\mathbf{1 0 0}$ \\
\hline
\end{tabular}

$68 \%$ indica que no le provoca incertidumbre y el $31 \%$ señala que si, un $1 \%$ omite

\section{Tabla $\mathbf{N}^{0} 1$ Desde qué punto de vista le provoca incertidumbre}

En relación a la pregunta si le provoca incertidumbre la jubilación, el $42 \%$ señala que ésta es desde el punto de vista económico y un $24 \%$ señala que es económico y llegar a la tercera edad, un $20 \%$ plantea que la incertidumbre es por llegar a la tercera edad, el $7 \%$ cree que es por la falta de actividad laboral, el $7 \%$ restante se distribuye en combinaciones con el factor económico.

Frente a la consulta si quiere jubilar, el $66 \%$ señala que si desea jubilar y el $33 \%$ restante señala que no desea jubilar.

Frente a la pregunta si quiere jubilar, desde el punto de vista del sexo del encuestado, se concluye que el $65 \%$ si quiere jubilar, y un $35 \%$ no quiere jubilar, de la cantidad de personas que si quieren jubilar se destaca que el $47 \%$ corresponde al sexo femenino y un $63 \%$ al sexo masculino.

\section{Tabla $\mathbf{N}^{\mathrm{a}} 2$ ¿Ud. quiere jubilar?, por escalafón}

\begin{tabular}{|l|c|c|c|}
\hline \multirow{2}{*}{ Le gustaría jubilar } & \multicolumn{2}{|c|}{ ESCALAFON } & \multirow{2}{*}{ Total } \\
\cline { 2 - 4 } & $\begin{array}{c}\text { Directivo- } \\
\text { Profesional }\end{array}$ & $\begin{array}{c}\text { Administrativo- } \\
\text { Técnico }\end{array}$ & \\
\hline $\mathrm{Si}$ & $15(63 \%)$ & $38(67 \%)$ & $53(65 \%)$ \\
\hline No & $9(38 \%)$ & $19(33 \%)$ & $28(35 \%)$ \\
\hline Total & $\mathbf{2 4}$ & $\mathbf{5 7}$ & $\mathbf{8 1}(\mathbf{1 0 0 \%})$ \\
\hline
\end{tabular}

En cuanto a si le gustaría jubilar, relacionado con el escalafón del individuo, podemos concluir que el $65 \%$ si quiere jubilar, del subtotal que quiere jubilar el $67 \%$, corresponde al escalafón Administrativo-Técnico y un 63\% al escalafón Directivo-Profesional. Un 35\% no quiere jubilar, en este subtotal un 33\% corresponde al escalafón Administrativo-Técnico y un $38 \%$ al escalafón Directivo-Profesional. 
Respecto a la pregunta del tiempo en que le gustaría jubilar, el 36\% señala que le gustaría jubilar al mismo tiempo que su pareja, un 32\% señala que le gustaría jubilar después que su pareja, un $30 \%$ le gustaría jubilar antes y al $2 \%$ le da lo mismo.

Respecto a la consulta que se les hizo a las personas que se encuentran en edad de jubilar, el $73 \%$ señala que aún no lo ha realizado porque si lo hiciera disminuiría su ingreso mensual de manera considerable y un $20 \%$ señala que no lo ha realizado porque le gusta seguir trabajando. El $7 \%$ no lo hace por ambas razones. El 79\% piensa que sí, que estará libre de exigencias y sólo el 20\% no lo cree, el $1 \%$ restante omite. Respecto a si con la jubilación dedicará más tiempo a aspectos de su vida que tenía postergados, el $84 \%$ señala que si lo hará y el $14 \%$ restante que no, el $2 \%$ restante omite. Al ser consultados en torno a si con la jubilación dará paso a una nueva etapa de su vida, el $62 \%$ así lo cree y un $38 \%$ cree que no.

En cuanto a si eventualmente podrá poner en marcha los planes que hace mucho tiempo tenía aplazados, el $72 \%$ indica que sí y el $27 \%$ no pondrán en marcha los planes que quería hacer, un $1 \%$ omite. Frente a la consulta de si la jubilación supondrá un cambio importante en su vida, el $80 \%$ lo confirma y el $19 \%$ lo desestima, el $1 \%$ omite.

\section{VARIABLE DE ESTUDIO 2: PREPARACIÓN PARA LA JUBILACIÓN EN RELACION A LAS DIMENSIONES:}

\section{A.- SALUD}

Referente a la afiliación del sistema de salud de los individuos, cabe destacar que el 65\% de éstos se encuentra afiliado a FONASA y el 35\% restante está afiliado a Isapre.

Respecto a la medicación que consumen al día se reporta que el 36\% de las personas consumen entre 3 a 4 medicamentos al día, el 28\% sólo 1 medicamento al día, el $25 \%$ solo 2 medicamentos al día y el $11 \%$ restante consume más de 5 medicamentos al día.

En cuanto a realizarse controles médicos periódicos por sus enfermedades, el $29 \%$ indica que lo realiza cada tres meses y el $26 \%$ lo realiza una vez al año, el otro $26 \%$ lo hace una vez al mes y el $18 \%$ restante lo realiza cada seis meses, un $1 \%$ omite. 


\section{B.- ECONOMIA}

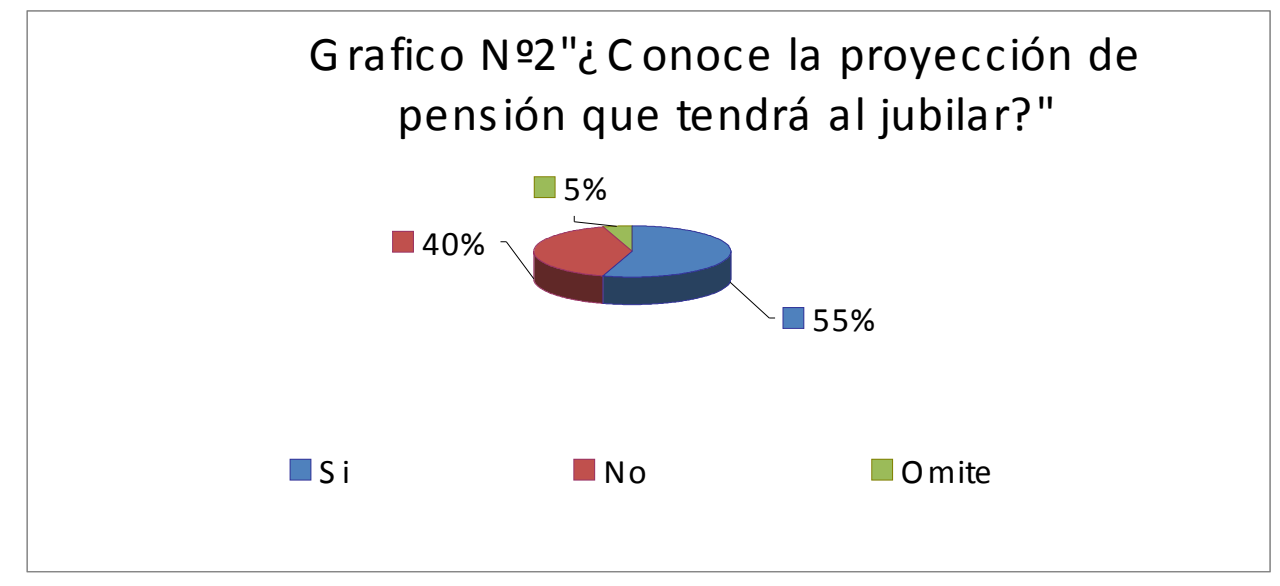

\section{Gráfico $\mathbf{N}^{\circ} 2$ Proyección de pensión al jubilar}

$\mathrm{Al}$ ser consultados en torno a si conocen la proyección de pensión que recibirán al jubilar, el 55\% indica que si la conoce y el $40 \%$ indica que la desconoce. Un 5\% omite responder.

Respecto a tener algún seguro de ahorro o si cotiza para el fondo APV en la AFP, el $80 \%$ señala que no y el $20 \%$ señala que si cotiza para un seguro de ahorro, el $13 \%$ corresponde al sexo femenino. Frente a la consulta si tiene algún trabajo remunerado adicional, se puede concluir que el $89 \%$ no lo tiene y de aquellos que sí lo poseen (11\%), el 10\% corresponde a sexo masculino y el $1 \%$ al sexo femenino.

\section{C.- VIVIENDA}

\section{Tenencia de la vivienda}

Referente a la situación actual de su vivienda, el $63 \%$ indica ser propietario y el $21 \%$ señala que es arrendada, en tanto 5\% es cedida y otro 5\% es sucesión, un $6 \%$ no especifica. En cuanto a si le parece adecuada su vivienda para vivir en ella, el $80 \%$ de las personas cree que si lo es y tan solo el $15 \%$ cree que no es adecuada, un $5 \%$ omite.

\section{Tabla $N^{\circ} 4$ La vivienda cuenta con pasamanos.}

Tabla $\mathbf{N}^{\circ}$ 4: En esto momentos su vivienda cuenta con algunos de estos elementos: pasamanos en la escalera, barras de sujeción en la ducha, antideslizantes en la tina $o$ en el piso.

\begin{tabular}{|l|c|c|}
\hline & Frecuencia & Porcentaje \\
\hline No & 59 & 70 \\
\hline $\mathrm{Si}$ & 24 & 29 \\
\hline Omite & 1 & 1 \\
\hline Total & $\mathbf{8 4}$ & $\mathbf{1 0 0}$ \\
\hline
\end{tabular}


Respecto a las condiciones de su vivienda, sólo el $29 \%$ de las personas posee en su vivienda algunos de éstos elementos tales como: pasamanos en la escalera, barras de sujeción en la ducha, antideslizantes en la tina o en el piso y el $70 \%$ no posee alguno de estos elementos. Un $1 \%$ omite respuesta.

\section{D.- REDES Y RELACIONES SOCIALES}

Tabla Nº 5 Relaciones con su cónyuge o pareja

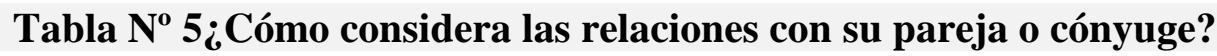

\begin{tabular}{|l|c|c|}
\hline & Frecuencia & Porcentaje \\
\hline Buenas & 31 & 37 \\
\hline Muy buenas & 27 & 32 \\
\hline No responde & 12 & 14 \\
\hline Regulares & 6 & 7 \\
\hline Malas & 4 & 5 \\
\hline No sabe & 2 & 3 \\
\hline Muy malas & 1 & 1 \\
\hline No tiene & 1 & 1 \\
\hline Total & $\mathbf{8 4}$ & $\mathbf{1 0 0}$ \\
\hline
\end{tabular}

En cuanto a las relaciones con su pareja o cónyuge, el $69 \%$ considera que son buenas o muy buenas, mientras que solo el $13 \%$ las considera muy malas, malas o regulares. Un 1\% no sabe y otro $1 \%$ no tiene pareja.

\section{Tabla $\mathbf{N}^{\circ} 6$ Relaciones con su cónyuge por sexo}

Tabla $\mathbf{N}^{0}$ 6: ¿Cómo considera las relaciones con su pareja o cónyuge, relacionado con el sexo

\begin{tabular}{|l|c|c|c|}
\hline Relaciones con su pareja & \multicolumn{2}{|c|}{ SEXO } & \\
& Masculino & Femenino & Total \\
\hline Muy buenas & $14(44 \%)$ & $11(30 \%)$ & $25(36 \%)$ \\
\hline Buenas & $13(41 \%)$ & $18(40 \%)$ & $31(45 \%)$ \\
\hline Regulares & $4(13 \%)$ & $1(1 \%)$ & $5(7 \%)$ \\
\hline Malas & $1(3 \%)$ & $3(4 \%)$ & $4(6 \%)$ \\
\hline Muy malas & 0 & $1(1 \%)$ & $1(1 \%)$ \\
\hline No sabe & 0 & $2(3 \%)$ & $2(3 \%)$ \\
\hline No tiene & 0 & $1(1 \%)$ & $1(1 \%)$ \\
\hline Total & $\mathbf{3 2 ( 4 6 \% )}$ & $\mathbf{3 7 ( 5 4 \% )}$ & $\mathbf{6 9}(\mathbf{1 0 0} \%)$ \\
\hline
\end{tabular}

En cuanto a las relaciones con su pareja o cónyuge, el $45 \%$ considera que son buenas, el $36 \%$ considera que las relaciones son muy buenas,. El $4 \%$ considera que son malas. 
Tabla $\mathbf{N}^{\circ} 7$ Relaciones con sus compañeros de trabajo

Tabla $\mathbf{N}^{0}$ 7: ¿Cómo son las relaciones con sus compañeros de trabajo?

\begin{tabular}{|l|c|c|}
\hline & Frecuencia & Porcentaje \\
\hline Buenas & 52 & 63 \\
\hline Muy buenas & 20 & 24 \\
\hline No sabe & 5 & 6 \\
\hline Regulares & 4 & 5 \\
\hline Malas & 1 & 1 \\
\hline Total & $\mathbf{8 2}$ & $\mathbf{1 0 0}$ \\
\hline
\end{tabular}

Respecto a las relaciones con sus compañeros de trabajo, se mantiene la misma tendencia que en las anteriores donde la mayoría señala que son buenas, $63 \%$, el $24 \%$ señala que son muy buenas, un 5\% indica que son regulares y tan sólo el $1 \%$ indica que son malas.

\section{Grafico $\mathbf{N}^{\circ} 4$ Cuenta con quien lo cuide}

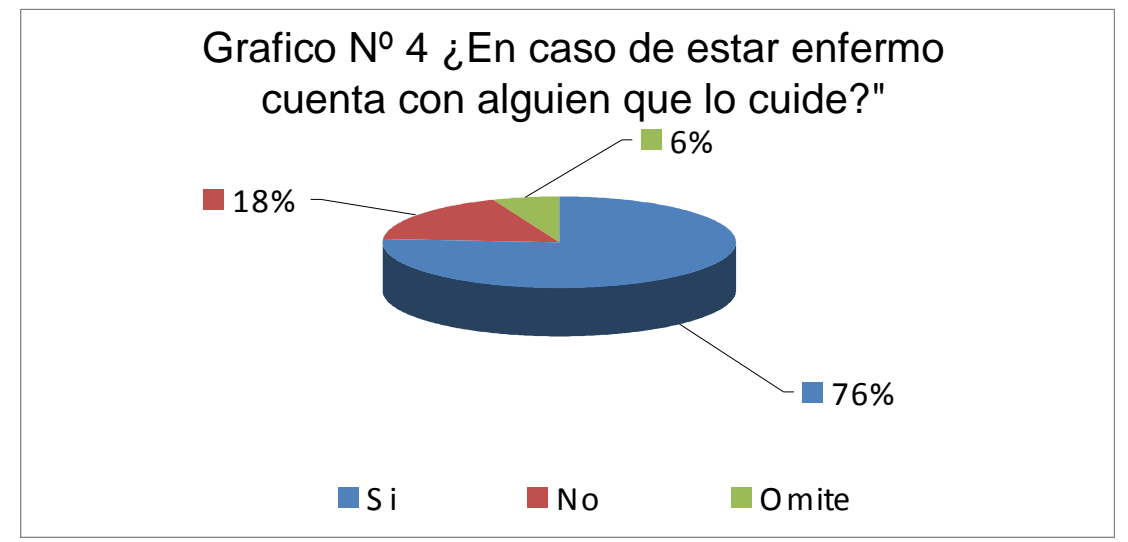

Frente a la pregunta si cuenta con alguien que lo cuide en la eventualidad de enfermedad, el $76 \%$ señala que si cuenta con un soporte y el $18 \%$ señala no poseer a nadie. Un $6 \%$ omite

En cuanto a la identificación de la persona que otorga los cuidados en estos casos, el 59\% señala que es la pareja o cónyuge quien lo realiza y el $13 \%$ refiere que esta tarea la cumple la hija, el resto señala más de una persona en distintas combinaciones.

En cuanto a la facilidad para hacer nuevas amistades, el $44 \%$ señala que regularmente, el $33 \%$ señala que totalmente y sólo el $16 \%$ señala que no tiene facilidad. Un $7 \%$ no sabe. En cuanto a la regularidad con que se relaciona con personas que no sean compañeros de trabajo, el $41 \%$ indica que lo hace una vez a la semana, el $16 \%$ señala que se relaciona con otras personas dos veces por semana, un $25 \%$ no lo hace, el resto de las personas lo hace con frecuencias mayores. 
Respecto a si participa en alguna organización fuera de su ámbito laboral, el $62 \%$ señala que no y el $31 \%$ indica que si pertenece a alguna organización, el $7 \%$ restante omite. De las personas que participan en alguna organización o grupo fuera de su ámbito laboral, el $36 \%$ indica que pertenece a otro grupo tales como: partidos políticos, talleres de artesanía, etc. siendo la participación masculina mayor en partidos políticos, juntas de vecinos y clubes deportivos y las mujeres en centros de padres, clubes de amigos y talleres de artesanía.

\section{E.- TIEMPO LIBRE}

\section{Planificación del tiempo libre}

Respecto a la planificación del tiempo libre, 54\% indica que no lo ha hecho y el $46 \%$ refiere que si lo ha planificado. En cuanto a la distribución por sexo, el $64 \%$ corresponde al sexo femenino y el $36 \%$ restante al masculino.

\section{Planificación tiempo libre por escalafón}

Relacionado a la planificación el tiempo libre una vez que jubile, con el escalafón laboral del individuo, se concluye que el $54 \%$ de las personas no lo ha planificado, no obstante del $46 \%$ que si lo ha planificado, el $43 \%$ corresponde al administrativo técnico y el $57 \%$ al directivo profesional. Frente a la pregunta si tiene algún pasatiempo, el $69 \%$ indica que si y el $31 \%$ señala que no tiene ninguno.

\section{Práctica de actividad física}

Respecto a la actividad física y si practica algún deporte, sólo el $35 \%$ alude a que si lo practica y un $65 \%$ no practica ningún deporte.

\section{Tipo de actividad física que realiza}

Consultado el universo estudiado, respecto si realizan actividad física, relacionado con el sexo, se destaca a un $44 \%$ que realiza caminatas, correspondiendo un $80 \%$ de ellos al sexo femenino y un $20 \%$ sexo masculino. Un $28 \%$ realiza ejercicios, distribuidos en un $60 \%$ sexo masculino y un $40 \%$ sexo femenino.

De las personas que contestaron que si realizan actividad física, relacionado con el escalafón se destaca a un $40 \%$ realiza caminatas, un $67 \%$ corresponde al escalafón Administrativo-Técnico y un 33\% al escalafón Directivo-Profesional. Un 33\% realiza ejercicios, distribuidos en un 50\% directivo profesional y un $50 \%$ escalafón administrativo técnico. En cuanto a la frecuencia con que realizan actividad física, el $35 \%$ lo realizan una vez por semana y el $29 \%$ dos a tres veces por semana como todos los días, los restantes lo hacen una vez al mes. 
Tabla $N^{\circ} 8$ Organización tiempo libre de 09:00 a 14:00 hrs

Tabla $\mathbf{N}^{0} 8$ Al jubilar, como utilizará su tiempo desde las 09:00 de la mañana hasta las 14:00 horas

\begin{tabular}{|c|c|c|}
\hline & Frecuencia & Porcentaje \\
\hline Lavar, cocinar y/o planchar & 22 & 26 \\
\hline Ayudar en las actividades de la casa & 21 & 28 \\
\hline $\begin{array}{l}\text { Ir a cancelar cuentas, ver tv, escuchar radio, visitar } \\
\text { amigos, caminar por el vecindario y ayudar en actividades } \\
\text { de la casa. }\end{array}$ & 5 & 6 \\
\hline Ir a cancelar cuentas & 4 & 5 \\
\hline Caminar por el vecindario & 4 & 5 \\
\hline Visitar a vecinos o amigos & 3 & 4 \\
\hline $\begin{array}{l}\text { Ver TV, escuchar radio y ayudar en las actividades de la } \\
\text { casa }\end{array}$ & 2 & 3 \\
\hline Ver TV, escuchar radio y caminar por el vecindario & 2 & 3 \\
\hline $\begin{array}{l}\text { Lavar, cocinar, planchar, ver tv, escuchar radio, visitar } \\
\text { amigos, caminar por el vecindario, ayudar en las } \\
\text { actividades de la casa. }\end{array}$ & 2 & 3 \\
\hline Ir a cancelar cuentas y ayudar en actividades de la casa. & 2 & 3 \\
\hline Ver TV, escuchar radio & 1 & 1 \\
\hline Lavar, cocinar, planchar, ver tv y escuchar radio. & 1 & 1 \\
\hline $\begin{array}{l}\text { Lavar, cocinar, planchar, ver tv y escuchar radio y ayudar } \\
\text { en las actividades de la casa. }\end{array}$ & 1 & 1 \\
\hline $\begin{array}{l}\text { Lavar, cocinar, planchar, ver tv y escuchar radio, ir a } \\
\text { cancelar cuentas y ayudar en las actividades de la casa. }\end{array}$ & 1 & 1 \\
\hline $\begin{array}{l}\text { Ver tv, escuchar radio, visitar amigos y ayudar en las } \\
\text { actividades de la casa. }\end{array}$ & 1 & 1 \\
\hline Lavar, cocinar y planchar e ir a cancelar cuentas. & 1 & 1 \\
\hline $\begin{array}{l}\text { Lavar, cocinar y planchar e ir a cancelar cuentas, ver tv y } \\
\text { escuchar radio. }\end{array}$ & 1 & 1 \\
\hline $\begin{array}{l}\text { Ir a cancelar cuentas, ver tv, escuchar radio y ayudar en } \\
\text { las actividades de la casa. }\end{array}$ & 1 & 1 \\
\hline Otras & 1 & 1 \\
\hline Ayudar en las actividades de la casa y otras. & 1 & 1 \\
\hline Total & 77 & 100 \\
\hline
\end{tabular}

Frente a la consulta en torno a cómo estructurará su rutina al momento de jubilar desde las 09:00 hasta las 14:00 horas, 57 personas señalaron que realizará tareas como: lavar, cocinar y /o planchar y ayudará en actividades de la casa, y solo 14 señala que participará en actividades sociales como visitar amigos, 10 personas omiten. 


\section{DISCUSIÓN}

En relación a la percepción frente a la jubilación, los encuestados respondieron según las diferentes percepciones:

Al $31 \%$ de los encuestados les provoca incertidumbre y ésta se relaciona con el factor económico en un $42 \%$ y el factor edad en un $24 \%$ lo cual demuestra que a un $66 \%$ de los encuestados la jubilación les provoca incertidumbre relacionada al factor económico y la edad.

Estos resultados guardan relación con el estudio entregado por la Superintendencia de AFP en el año 2004, que dice que los jubilados por el sistema de capitalización individual verán mermados sus ingresos. El 89\% señaló que este período represente un merecido descanso siendo el porcentaje mayor el administrativo técnico (91\%) por sobre el directivo profesional (83\%)

Según la literatura los resultados obtenidos confirman lo expresado por Atchley (7) en cuanto a las diferentes fases de la transición a la jubilación que experimentarían los individuos, si bien es cierto en este caso, no serian fases sino diferentes formas alternativas de percibir la jubilación. La primera de ellas, es percibirla como un descanso y alivio y la segunda como un nuevo comienzo lleno de oportunidades y de mayor libertad. En tercer lugar, la vivencian como pérdida de actividades y roles.

En cuanto al rechazo y preocupación demostrado por el sexo femenino, esto estaría explicado por la investigadora Raquel Malla (8), particularmente cuando la mujer es jefa de hogar o trabaja por necesidad económica, situación en que la jubilación se afronta con angustia porque supone una reducción importante de los ingresos. Cuando la mujer desarrolla un trabajo que le satisface, las pérdidas que supone la jubilación son similares a las del hombre y de allí también el rechazo.

Además, se puede establecer que frente a esta diversidad de percepciones, el significado que la jubilación pueda tener para la mujer y el hombre, depende de la importancia que cada uno le asigne al trabajo que desempeña Castaño y Martínez (1990).

\section{En relación a la preparación de la jubilación}

\section{A.- Salud}

El $100 \%$ de los encuestados cuenta con un sistema de protección de salud ya sea FONASA con un $65 \%$ o con ISAPRE en un $35 \%$.

\section{B.- Económicos}

El $50 \%$ se encuentra adscrito a AFP, un 50\% en INP, se observa que en materia previsional coexisten dos sistemas previsionales que otorgan diferencias en cuanto a montos de pensión. A contar de 1983 todo trabajador debe estar afiliado a una AFP, pero como los encuestados ingresaron antes de esta fecha encontramos a personas en el sistema antiguo. 
Lo que sorprende es que el $40 \%$ no conoce su proyección de pensión a futuro, y además no tienen ahorro un $73 \%$ lo que, contrastado con el último informe de la superintendencia de AFP, indica que los futuros pensionados verán una merma de sus ingresos. Teniendo en cuenta lo anterior se prevé la siguiente situación: estos funcionarios (as) accederán a pensiones mínimas, lo cual incidirá negativamente en sus ingresos y por ende en la satisfacción de sus necesidades, esto concuerda con el último informe de la Súper Intendencia de AFP 2004. En cambio los beneficiarios del INP tendrán una pensión similar al ingreso que actualmente perciben.

\section{C.- Vivienda}

En cuanto a la tenencia de la vivienda el $63 \% \%$ es dueño de su casa los resultados son concordantes con la encuesta CASEN 2003 lo cual denota la cultura del bien raíz como principal medida de preparación para la jubilación, solo el $35 \%$ desearía cambiarse cuando jubile pero, señalaron en un 59\%, que los medios económicos lo impedían. El 80\%, encuentra sus casas adecuadas para vivir en ellas sin embargo, un $70 \%$ señaló que estas viviendas no cuentan con medidas de protección para prevenir caídas u otros accidentes.

Si consideramos que la dependencia /enfermedad se asocia a la edad, es decir a mayor edad mayor dependencia y que la expectativa de vida en Chile ha aumentado sobre todo en el grupo de 70 años y mas, la preparación para la jubilación, desde el punto de vista arquitectónico, no ha previsto un hogar libre de barreras y con seguridad contra accidentes. Desde el punto de vista social, ellos prefieren quedarse en sus viviendas y no se cambiarían de domicilio para estar cerca de amigos en un $90 \%$.

\section{D.- Redes y Relaciones sociales}

El $69 \%$ considera que sus relaciones de pareja son satisfactorias al igual que con sus hijos en un $81 \%$, con los compañeros de trabajo en un $87 \%$, manteniendo buenas relaciones con sus vecinos en un $85 \%$.El $22 \%$ de los hombres no se relaciona nunca con personas ajenas a su trabajo y un $27 \%$ de las mujeres tampoco lo hace. Este dato es importantísimo desde el punto de vista de relaciones sociales considerada como factor protector de la adaptación a la jubilación.

El $83 \%$ señala que su familla es la principal fuente de apoyo siendo el cónyuge en primer lugar y luego la hija, quien los cuidaría en caso de estar enfermos. Lo que concuerda con lo señalado con Moragas en su libro sobre la jubilación.

Un $76 \%$ de los adultos mayores, cuenta con la familia como principal apoyo. Un $18 \%$ no cuenta con apoyo para la situación de enfermedad, lo cual sumado al $23 \%$ que no se relaciona con amistades fuera de su ámbito laboral y familiar, convierte a estos sujetos en un grupo vulnerable en cuanto a redes se refiere, ante la soledad y el abandono.

En cuanto a la participación social, solamente el 31\% participa en alguna organización grupal fuera de su ámbito de trabajo, lo que limita el contacto social con nuevos grupos de amigos. Dentro de la participación, la mayoría lo hace en actividad política y talleres de artesanía. Siendo más alta esta participación en el sexo masculino (45\%) por sobre el sexo femenino (28\%). La 
participación social es mayor en hombres y ésta se da más en grupos políticos, mientras que las mujeres prefieren el grupo de amigas.

En relación a otro de los factores protectores mencionados en nuestro marco conceptual como son las relaciones sociales, el estudio arrojó como resultado que los hombres consideran mayoritariamente buenas o muy buenas sus relaciones con su pareja (85\%) en cambio las mujeres las consideran buenas o muy buenas en un (70\%).

En cuanto a la frecuencia de relaciones con personas que no son compañeros de trabajo, el estudio arrojó que el $33 \%$ de las mujeres se relaciona sólo una vez por semana con personas fuera del ámbito laboral, el $20 \%$ de las mujeres se relaciona dos veces por semana, el 10\% de las mujeres tres veces por semana y el $4 \%$ de éstas cuatro veces por semana. Sin embargo hay que destacar que el $27 \%$ de las mujeres no se relaciona ninguna vez con personas que no sean compañeros de trabajo. Lo cual demuestra la falencia de relaciones por frecuencia que presenta el grupo de mujeres. En los hombres el $53 \%$ lo hace una vez por semana y el $21 \%$ ninguna vez. La situación por ende es más crítica en los hombres.

\section{E.- Tiempo libre}

En relación con la planificación del tiempo libre, no se aprecian diferencias considerables ya que solo un $54 \%$ lo tiene planificado, en cambio un $46 \%$ no lo tiene planificado, un $30 \%$ no tiene pasatiempo contra un $66 \%$ que si lo tiene.

En cuanto a la actividad física, un $65 \%$ no realiza actividad física y un $87 \%$ no practica ningún deporte lo cual guarda relación con la encuesta SABE y la Encuesta calidad de Vida 2000. Al preguntar como planificaría un día, los datos arrojan que en un $26 \%$ realizarán actividades como lavar, planchar, asear y el otro $25 \%$ señala cooperar en ellas. Lo cual guarda relación con la revisión de la literatura en el sentido que la mujer debe adaptarse a su nuevo papel en el hogar, una vez superadas la mayor parte de sus obligaciones laborales.

La dedicación a las obligaciones hogareñas sufre un incremento, viene a sustituir parte del trabajo asalariado fuera del hogar por el trabajo en casa. La mujer vuelve a la continuación de su rol como ama de casa... En cambio en los hombres el 29\% señaló que ayudará en las actividades de la casa y solo un 7\% realizará las tareas de lavar, cocinar y asear. Como señala Raquel Malla, el hombre se instala en su casa y su asunción de tareas supone para la mujer construir una nueva forma de convivencia, la responsabilidad de la buena marcha de la vida doméstica sigue siendo de la mujer.

\section{MEDIDAS PROPUESTAS}

En relación a los resultados de la investigación y a las conclusiones presentadas, se proponen las siguientes medidas:

\section{A) Talleres de Preparación Cognitiva, Psíquica y Física.}


Los y las funcionarias en un $61 \%$ están dispuestos a participar en un curso de preparación para la jubilación. Por tanto INP como ente ejecutor de las políticas del supremo gobierno en materia de previsión, AFP como órgano de administración privado, CCAF, Mutualidades deberían entregar estos cursos a través de aportes en los cuales hubiese participación de los entes previsionales involucrados y las empresas.

La preparación para la jubilación es necesaria y debe realizarse unos 7 a 5 años antes de que la persona cumpla la edad para jubilar. Debe ser un proceso que involucre vencer los estigmas negativos que la jubilación y que la vejez imponen.

Esta preparación para la jubilación deberá incorporar los duelos y también a la pareja u otro miembro del grupo familiar. Deberá considerar los factores físicos, psíquicos y cognitivos. Por lo que el Terapeuta Ocupacional debe diseñar y ejecutar los diferentes talleres que ayuden en esta transición ocupacional de acuerdo a sus capacidades:

\section{B) Salud}

Se propone la implementación de un chequeo o examen médico obligatorio al término de la vida laboral, similar al realizado al inicio de ella, en la administración pública, con el fin de que la persona tome las prevenciones necesarias en la nueva etapa que comienza.

\section{C) en Relación a Redes de Apoyo}

Fomentar las relaciones con el otro y la cooperación entre el grupo de amigos y vecindario con el objeto de potenciar las redes sociales.

\section{D) En Relación a Información:}

Mejorar canales de información en materia de actividades para ocupar el tiempo libre, talleres de formación, de entretención, voluntariado. Fomentar la participación social en los adultos mayores en términos de autonomía.

\section{E) Adaptación a la Nueva Etapa}

Flexibilidad en horario para aquellas personas que cumplen la edad para la jubilación. Reducir la jornada de trabajo a jornada parcial por el lapso de 6 meses, tiempo que puede demorar su jubilación, con el fin de adaptarlos para la realización de actividades de ocio y prepararlos psicológicamente en la desvinculación al medio laboral.

\section{F) Mayores Recursos Profesionales y Administrativos Capacitados}

Mayor profesionalización en materias de adultos mayores. 


\section{REFERENCIAS BIBLIOGRÁFICAS}

1. INE. (2004). Chile y los adultos mayores, impacto en la sociedad del 2000 . Santiago.

2. GÓMEZ, S. Equilibrio y organización de la rutina diaria Revista Chilena de Terapia Ocupacional, 2009. Consultado el mar 22, 2011, de http://www.revistas.uchile.cl/index.php/RTO/article/viewArticle/111/96

3. MORAGAS, R. (2000). La jubilacion un enfoque positivo. Grifjalgo.

4. MORALES, M. (2001, Vol VII , $\mathrm{N}^{\circ}$ 1). Los adultos mayores chilenos en el siglo XX: un enfoque politologico. Acta Bioética .

5. RAMOS, M. (2001). Antropologia de la vejez desde la perspectiva de genero

6. HERNÁNDEZ, R., FERNÁNDEZ, C., \& BAPTISTA, P. (2006). Definición del alcance de la investigación a realizar: exploratoria, descriptiva, correlacional o explicativa. En R. Hernández, C. Fernández, \& P. Baptista, Metodología de la Investigación (págs. 100102). México D.F.: Mc Graw Hill.

7. AGULLO, T., \& SILVERIO, M. (2001). Mayores actividad y trabajo: una aproximacion psicosociologica. Madrid: Ministerio del trabajo y asuntos sociales España.

8. ATCHLEY, R.C. (1979). Issues in retirement research. The Gerontologist, 19

9. MALLA, R. (2001) Mujer, jubilación y ocio. Rev. Mult. Gerontol. 11(4): 186-188

10. CASTAÑO, D.; MARTÍNEZ-BENLLOCH, I. (1990) Aspectos psicosociales del envejecimiento de las mujeres. Anales de psicología, 6 (2), 159-168. 